Для цитирования: Хамина В.В. Особенности адаптационных возможностей у больных шизофренией с сопутствующим метаболическим синдромом. Сибирский вестник психиатрии и наркологии. 2020; 3 (108): 51-56. https://doi.org/10.26617/1810-3111-2020-3(108)-51-56

\title{
Особенности адаптационных возможностей у больных шизофренией с сопутствующим метаболическим синдромом
}

\author{
Хамина В.В. \\ НИИ психического здоровья, Томский национальньй исследовательский медицинский цฺентр \\ Российской академии наук \\ Россия, 634014, Томск, ул. Алеутская, 4
}

\begin{abstract}
PEЗЮME
Актуальность работы детерминируется востребованностью исследований по уточнению и конкретизации адаптационного потенциала при шизофрении с сопутствующим метаболическим синдромом (МС) и необходимостью оценки его взаимосвязи с клинико-конституциональными особенностями пациентов. Цель: изучить особенности адаптационных возможностей пациентов с шизофренией в зависимости от наличия или отсутствия сопутствующего МС. Материал и методы. Было обследовано 150 пациентов. Диагноз шизофрении (F20) ставился с учетом критериев МКБ-10. Диагноз МС верифицирован в соответствии с критериями Международной Федерации диабета (International Diabetes Federation, 2005). Оценку адаптации больных шизофренией проводили с помощью методики Г.В. Логвинович с определением типа компенсаторно-приспособительной защиты пациентов и типа адаптации. Психометрическая оценка клинического состояния проводилась с использованием шкалы PANSS. Обсуждение: пациенты с шизофренией и МС склонны, так же как и пациенты без МС, в большинстве случаев формировать тип компенсаторно-приспособительной защиты в виде «укрытия под опекой». Среди типов адаптации у больных шизофренией и сопутствующим МС преобладают неблагоприятные (деструктивный и экстравертный). Тяжесть клинического состояния в психометрическом выражении по PANSS, а также особенности конституции у пациентов с наличием сопутствующего МС в зависимости от типа социальной адаптации не отличаются.
\end{abstract}

Ключевые слова: шизофрения, метаболический синдром, типы адаптации, типы компенсаторноприспособительной защиты, шкала PANSS.

\section{ВВЕДЕНИЕ}

Шизофрения - социально значимое заболевание современного общества, характеризующееся хроническим течением и приводящее к инвалидизации больных в результате прогрессирования психических нарушений и развивающихся соматических осложнений, а также сочетанной соматической патологии вследствие генетических и биологических факторов риска, нередко связанных с терапией основного расстройства $[1,2,3]$. Одним из наиболее часто встречающихся сочетанных соматических состояний, наблюдающихся при шизофрении, является $\mathrm{MC}$, а ведущее место среди причин смерти пациентов с шизофренией на сегодняшний день занимают сердечнососудистые расстройства $[4,5,6]$.

В совокупный состав МС входят артериальная гипертензия, нарушение толерантности к глюкозе, ишемическая болезнь сердца, сахарный диабет второго типа и сопутствующие этим заболеваниям гормональные и метаболические расстройства $[7,8,9]$. У больных шизофренией МС часто является фактором, отягощающим течение основного расстройства [10]. Клинические проявления шизофрении также оказывают влияние на компоненты МС [11].
Так, у больных с выраженной негативной симптоматикой и ассоциированным с ней малоподвижным образом жизни чаще встречаются нарушения липидного обмена [12, 13]. Однако влияние негативных симптомов на вес пациентов остается дискутабельной темой: например, некоторые исследования демонстрируют его снижение, другие, напротив, повышение [12]. В соответствии с последним зарубежным метааналитическим обзором около трети пациентов с шизофренией страдают МС [14], а по данным российских исследователей его распространенность составляет $35,5 \%$ [15].

Наличие МС у психически здоровых лиц в основном ухудшает адаптационные возможности, нередко привнося расстройства астенического (колебания настроения, вегетативная лабильность, повышенная утомляемость, расстройства сна, снижение работоспособности) и тревожного (повышенный уровень личностной тревоги, ипохондрические фиксации) круга [16]. Социальное функционирование больных шизофренией и сопутствующим МС часто снижается [17], однако влияние метаболических нарушений на возможности адаптации изучено недостаточно. 
Таким образом, актуальность данного исследования определяется необходимостью уточнить и конкретизировать адаптационные особенности при шизофрении с сопутствующим $\mathrm{MC}$, а также оценить их взаимосвязь с клиникоконституциональными особенностями пациентов.

\section{ЦЕЛЬ ИССЛЕДОВАНИЯ}

Изучить особенности адаптационных возможностей пациентов с шизофренией в зависимости от наличия или отсутствия сопутствующего МС.

\section{МАТЕРИАЛЫ И МЕТОДЫ}

В исследование были включены 150 пациентов с шизофренией (F20.0-20.9 в соответствии с критериями МКБ-10). Возраст испытуемых составил от 18 до 60 лет. Все пациенты были госпитализированы в отделение эндогенных расстройств НИИ психического здоровья Томского НИМЦ и ОГАУЗ «Томская клиническая психиатрическая больница» в течение 6 недель. Больные дали письменное добровольное информированное согласие на участие в исследовании. Все пациенты основной группы были разделены на две подгруппы в зависимости от наличия или отсутствия MC. Диагноз МC верифицировался в соответствии с критериями Международной Федерации диабета (International Diabetes Federation - IDF, 2005) [18]. Согласно данным критериям при постановке диагноза МС учитываются следующие параметры: окружность талии, концентрации триглицеридов, липопротеинов высокой плотности, глюкозы, а также уровень артериального давления.

Оценку адаптации больных шизофренией проводили с помощью методики Г.В. Логвинович, заключающейся в определении типа компенсаторно-приспособительной защиты пациентов и типа адаптации [19]. Психометрическая оценка клинического состояния проводилась с использованием шкалы PANSS [20] в адаптированной русской версии [21]. Определение антропометрических показателей костной компоненты проводилось по методике В.В. Бунака (1941), разработанной в НИИ антропологии им. Д.Н. Анучина МГУ им. М.В. Ломоносова [22].

Для статистической обработки полученных данных использовали пакет программ для ПК «Statistica» (версия 10.0).

\section{РЕЗУЛЬТАТЫ И ОБСУЖДЕНИЕ}

Из 150 обследованных МС был выявлен у 63 пациентов (42\%) соответственно, у 87 пациентов (58\%) было зарегистрировано его отсутствие. Медиана возраста в подгруппе больных с сопутствующим МС составила $40[33 ; 52]$ лет. Среди больных без МС данный показатель составил 34 [27; 43] года. Следовательно, между исследуемыми подгруппами имелись статистически значимые отличия по возрасту ( $\mathrm{p}=0,0008)$.
Выявлено, что в подгруппе больных шизофренией и сопутствующим МС, как и в подгруппе без $\mathrm{MC}$, преобладал относительно неблагоприятный тип компенсаторно-приспособительной защиты «укрытие под опекой»: 38 (60,32\%) пациентов и $48(55,17 \%)$ соответственно. Наименее часто в обеих подгруппах больных шизофренией встречался тип защиты «социальная оппозиция»; в связи с малочисленностью данные подгруппы не сравнивались между собой: в подгруппе с сопутствующим $\mathrm{MC}-3$ человека $(4,76 \%)$, в подгруппе без МC -8 человек $(9,19 \%)$. Благоприятный тип компенсаторно-приспособительной защиты «гуттаперчевая капсула» в подгруппе с сопутствующим МС выявлен у 13 (20,63\%), в подгруппе пациентов с отсутствием - МС у 14 (16,09\%). Тип защиты «экологическая ниша» наблюдался в подгруппе с МС у 9 (14,29\%) пациентов, в подгруппе без сопутствующего МC - у 17 (19,54\%) пациентов. Статистически значимых различий в соотношении всех трех типов защит между больными шизофренией с учетом наличия или отсутствия МС обнаружено не было ( $>0,05)$.

Далее было изучено распределение больных шизофренией с отсутствием или наличием МC в зависимости от типа социальной адаптации: 1) относительно благоприятные (интравертный и интегративный), 2) относительно неблагоприятные (деструктивный и экстравертный).

В подгруппе пациентов с шизофренией без МC наблюдались относительно более компенсированные типы адаптации, в рамках которых сохранялось функционирование в микросоциальной и трудовой сферах. Так, интегративный тип социальной адаптации в данной подгруппе выявлен примерно у половины пациентов ( $\mathrm{n}=32,50,8 \%)$, затем по частоте встречаемости следовал интравертный тип - 26 (41,3\%), самое меньшая доля пациентов наблюдалась с экстравертным типом $(\mathrm{n}=5,7,9 \%)$. Деструктивный тип социальной адаптации, для которого характерна декомпенсация как на социальном, так и на клиническом уровне, у больных шизофренией обнаружен не был. В подгруппе больных шизофренией с MC отсутствовали оптимально скомпенсированные типы адаптации интегративного уровня; в этой подгруппе преобладали пациенты с экстравертным типом адаптации $(\mathrm{n}=48,55,2 \%)$, примерно у трети пациентов $(\mathrm{n}=26,29,9 \%)$ был сформирован деструктивный тип социальной адаптации, интравертный тип встречался с относительно редкой частотой $(\mathrm{n}=13,14,9 \%)$.

Была проведена оценка у больных шизофренией с учетом отсутствия/наличия $\mathrm{MC}$ количества баллов по шкале PANSS, значений индексов ReesEysenk и Tanner. Выполнен сравнительный анализ полученных данных с учетом благоприятных или 
неблагоприятных типов социальной адаптации. $\quad$ Результаты представлены в таблице 1.

Т а б л и ц а 1. Распределение показателей длительности течения заболевания, оценки баллов по шкале PANSS, значений индексов Rees-Eysenk и Tanner у больных шизофренией с отсутствием или наличием МС с учетом типа социальной адаптации

\begin{tabular}{|c|c|c|c|c|c|c|}
\hline \multirow{3}{*}{ Показатель } & \multicolumn{3}{|c|}{ Пациенты с МС } & \multicolumn{3}{|c|}{ Пациенты без МС } \\
\hline & \multicolumn{2}{|c|}{ Типы адаптации } & \multirow[t]{2}{*}{$\mathrm{p}$} & \multicolumn{2}{|c|}{ Типы адаптации } & \multirow{2}{*}{$\mathrm{p}$} \\
\hline & благоприятные & неблагоприятные & & благоприятные & неблагоприятные & \\
\hline $\begin{array}{c}\text { Длительность } \\
\text { заболевания }\end{array}$ & $20[15 ; 22]$ & $16[10 ; 22]$ & 0,414 & $8,5[4 ; 17]$ & $15[12 ; 27,5]$ & $0,041^{*}$ \\
\hline $\begin{array}{c}\text { PANSS позитивные } \\
\text { симптомы }\end{array}$ & $21[16 ; 23]$ & $20[17 ; 25]$ & 0,969 & $19[14 ; 22,5]$ & $21[17,5 ; 23]$ & 0,332 \\
\hline $\begin{array}{c}\text { PANSS негативные } \\
\text { симптомы }\end{array}$ & $27[25 ; 28]$ & $27[21 ; 31]$ & 0,738 & $24[21 ; 27,5]$ & $26[23 ; 29.5]$ & 0,356 \\
\hline $\begin{array}{c}\text { PANSS общие } \\
\text { психопатологиче- } \\
\text { ские симптомы }\end{array}$ & $54[50 ; 54]$ & $51[44 ; 56]$ & 0,529 & $47[41 ; 52]$ & $56[51 ; 59]$ & $0,047 *$ \\
\hline PANSS общий балл & $107[102 ; 109]$ & $100,5[92 ; 109]$ & 0,415 & $91,5[81 ; 99,5]$ & $104[97 ; 110,5]$ & $0,044 *$ \\
\hline Индекс Tanner & $97[96 ; 105]$ & $91[87 ; 98]$ & 0,101 & $91[84,5 ; 96]$ & $90[85,5 ; 95,3]$ & 0,950 \\
\hline Индекс Rees-Eysenk & $95[90,9 ; 105,4]$ & $97,7[93,3 ; 102,2]$ & 0,724 & $105[99,7 ; 109,2]$ & $\begin{array}{c}111,1[103,2 \\
115,2]\end{array}$ & 0,149 \\
\hline
\end{tabular}

П р и м е ч а н и е. Уровень значимости - p; *-статистически значимый уровень $\mathrm{p}<0,05$.

В противоположность подгруппе больных шизофренией с наличием сопутствующего МС у пациентов без МС три ведущих показателя, таких как длительность течения заболевания $(\mathrm{p}=0,041)$, количество баллов по шкале общих психопатологических симптомов по PANSS $(\mathrm{p}=0,047)$, а также общий балл по PANSS ( $\mathrm{p}=0,044)$, имели статистически значимо относительно более высокие значения среди больных с неблагоприятными типами адаптации по сравнению с больными с благоприятными типами.

\section{ЗАКЛЮЧЕНИЕ}

Пациенты с шизофренией и МС склонны, как и пациенты без МС, в большинстве случаев формировать тип компенсаторно-приспособительной защиты в виде «укрытия под опекой». В роли «опекуна» обычно выступают родственники больного, с которыми он проживает, либо работники психиатрических служб. Факторами, предположительно способствующими такому поведенческому стереотипу, являются негативные расстройства и когнитивные нарушения, сформированные в результате течения болезни [23, 24].

Среди типов адаптации у больных шизофренией и сопутствующим МС преобладают неблагоприятные (деструктивный и экстравертный). Тяжесть клинического состояния в психометрическом выражении по PANSS, а также особенности конституции у пациентов с наличием сопутствующего МС в зависимости от типа социальной адаптации не отличаются.

\section{КОНФЛИКТ ИНТЕРЕСОВ}

Автор заявляет, что представленные в статье материалы не имеют оснований для реального или потенциального конфликта интересов.

\section{ИСТОЧНИК ФИНАНСИРОВАНИЯ}

Исследование выполнено при поддержке гранта РНФ № 18-15-00011 «Шизофрения, сочетанная c метаболическим синдромом: клиникоконституциональные факторы и молекулярные маркеры».

\section{СООТВЕТСТВИЕ ПРИНЦИПАМ ЭТИКИ}

Настоящее исследование было одобрено локальным этическим комитетом НИИ психического здоровья Томского НИМЦ (протокол ЛЭК от 17.04.2017 № 99) и проводилось в соответствии с принципами Хельсинской декларации (2000).

\section{ЛИТЕРАТУРА/REFERENCES}

1. Piotrowski P.A., Gondek T.M., KrolickaDerkgowska A., Misiak B., Adamowski T., Kiejna A. Causes of mortality in schizophrenia: An updated review of European studies. Psychiatr Danub. 2017;29 (2): 108-120. doi: 10.24869/psyd.2017.108

2. Меднова И.А., Серебров В.Ю., Байков А.Н., Бохан Н.А., Иванова С.А. Аминокислоты и ацилкарнитины как потенциальные метаболомные маркеры шизофрении: новые подходы к диагностике и терапии. Бюллетень сибирской медицины. 2019; 18(4): 197-208. Mednova I.A., Serebrov V.Yu., Baikov A.N., Bokhan N.A., Ivanova S.A. Amino acids and acylcarnitines as potential metabolomic markers of schizophrenia: new approaches to diagnostics and therapy. Bulletin of Siberian Medicine. 2019;18(4):197-208 (in Russian). https://doi.org/10.20538/1682-0363-2019-4-197-208

3. Корнетова Е.Г., Семке А.В., Корнетов А.Н., Иванова С.А., Лобачева О.А., Семенюк К.А., Бойко А.С., Бохан Н.А. Шизофрения: биопсихосоциальная модель и конституциональнобиологический подход. Томск : ООО «Интегральный Переплет», 2018: 174. Kornetova E.G., 
Semke A.V., Kornetov A.N., Ivanova S.A., Lobacheva O.A., Semenyuk K.A., Boyko A.S., Bokhan N.A. Schizophrenia: a biopsychosocial model and a constitutional-biological approach. Tomsk: "Integral Cover", 2018: 174 (in Russian).

4. Волков В.П. Метаболический синдром: история вопроса. Universum: медицина и фармакология. 2017; 4 (38): 36-45. Volkov V.P. Metabolic syndrome: history of the issue. Universum: Medicine and Pharmacology. 2017; 4 (38): 36-45 (in Russian).

5. Dieset I., Andreassen O.A., Haukvik U.K. Somatic comorbidity in schizophrenia: some possible biological mechanisms across the life span. Schizophr. Bull. 2016; 42: 1316-1319. https://doi.org/10.1093/schbul/sbw028

6. Корнетова Е.Г., Меднова И.А., Дубровская В.В., Бойко А.С., Иванова С.А., Семке А.В. Метаболический синдром у больных шизофренией в психиатрической практике: Пособие для врачей. Томск, 2018: 32. Kornetova E.G., Mednova I.A., Dubrovskaya V.V., Boyko A.S., Ivanova S.A., Semke A.V. Metabolic syndrome in patients with schizophrenia in psychiatric practice: A guide for doctors. Tomsk, 2018: 32. (in Russian).

7. Агабабян И.Р., Джаббарова Н.М., Рофеев М.Ш., Назарова З.Ш., Пулатова К.С. Метаболический синдром как один из основных факторов развития артериальной гипертонии. Достижения науки и образования. 2019; 10 (51): 54-58. Agababyan I.R., Dzhabbarova N.M., Rofeev M.Sh., Nazarova Z.Sh., Pulatova K.S. Metabolic syndrome as one of the main factors in the development of arterial hypertension. Achievements of Science and Education. 2019; 10 (51): 54-58 (in Russian).

8. Дубровская В.В., Корнетова Е.Г., Семке А.В., Иванова С.А., Аржаник М.Б., Головаха Н.Э. Клиническая и социальная адаптация у пациентов с шизофренией и сопутствующим метаболическим синдромом. Современная терапия психических расстройств. 2019; 2: 17-21. Dubrovskaya V.V., Kornetova E.G., Semke A.V., Ivanova S.A., Arzhanik M.B., Golovakha N.E. Clinical and social adaptation in patients with schizophrenia and concomitant metabolic syndrome. Modern Therapy of Mental Disorders. 2019; 2: 17-21 (in Russian).

9. Бородюк Ю.Н., Дмитриева Е.Г., Корнетова Е.Г., Лобачева О.А., Смирнова Л.П., Паршукова Д.А., Козлова С.М., Семке А.В. Особенности адаптации больных параноидной шизофренией при нейролептической терапии с риском развития метаболического синдрома (изменение показателей липидного обмена сыворотки крови). Сибирский вестник психиатрии и наркологии. 2015; 2(87): 86-91. Borodyuk Yu.N., Dmitrieva E.G., Kornetova E.G., Lobacheva O.A., Smirnova L.P., Parshukova D.A., Kozlova S.M., Semke A.V. The adaptation features of patients suffering from paranoid schizophrenia and taking neuroleptics as medication with risk of development of the metabolic syndrome (with changed lipid metabolism indicators of blood serum). Siberian Herald of Psychiatry and Addiction Psychiatry. 2015; 2(87): 8691 (in Russian).

10. Bioque M., Garcia-Portilla M.A.P., Garcia-Rizo C., Cabrera B., Lobo A., Gonzalez-Pinto A., DiazCaneja C.M., Corripio I., Vieta E., Castro-Fornieles J., Bobes J., Gutierrez-Fraile M., RodriguezJimenez R., Mezquida G., Llerena A., Saiz-Ruiz J., Bernardo M. Evolution of metabolic risk factors over a two-year period in a cohort of first episodes of psychosis. Schizophr Res. 2018; 193: 188-196. doi: 10.1016/j.schres.2017.06.032

11. Saatcioglu O., Kalkan M., Fistikci N. Relationship between metabolic syndrome and clinical features, and its personal-social performance in patients with schizophrenia. Psychiatr Quarterly. 2016; 87(2): 265-280. doi: 10.1007/s11126-015-9384-0

12. Chen S.F., Hu T.M., Lan T.H. Severity of psychosis syndrome and change of metabolic abnormality in chronic schizophrenia patients: severe negative syndrome may be related to a distinct lipid pathophysiology. Eur Psychiatry. 2014; 29 (3): 167-71. doi: 10.1016/j.eurpsy.2013.04.003

13. Корнетова Е.Г., Семке А.В. Современные вопросы и перспективы изучения шизофрении с ведущей негативной симптоматикой. Бюллетень сибирской медицины. 2014;13 (1): 5-13. Kornetova E.G., Semke A.V. Modern issues and prospects of studying schizophrenia with leading negative symptoms. Bulletin of Siberian Medicine. 2014; 13 (1): 5-13 (in Russian).

14. Mitchell A.J., Vancampfort D., Sweers K., van Winkel R., Yu.W., De Hert M. Prevalence of metabolic syndrome and metabolic abnormalities in schizophrenia and related disorders - a systematic review and meta-analysis. Schizophrenia Bulletin. 2013; 39(2): 306-318. doi: 10.1093/schbul/sbr148

15. Незнанов Н.Г., Мартынихин И.А., Танянский Д. А., Ротарь О.П., Солнцев В. Н., Соколян Н.А., Денисенко А. Д. Шизофрения - фактор, увеличивающий риск развития метаболического синдрома. Результаты исследования с использованием метода подбора пар. Медицинский академический журнал. 2013; 13(3): 90-96. Neznanov N.G., Martynikhin I.A., Tanyansky D.A., Rotar O.P., Solntsev V.N., Sokolyan N.A., Denisenko A.D. Schizophrenia is a factor that elevates the risk of metabolic syndrome development. Results of the study using the matching method. Medical Academic Journal. 2013; 13 (3): 90-96 (in Russian).

16. Хохлов А.Л., Жилина А.Н., Буйдина Т.А. Взаимосвязь показателей качества жизни и особенностей психологического статуса с клиническими проявлениями метаболического синдрома. Качественная клиническая практика. 2006; 2: 19-23. Khokhlov A.L., Zhilina A.N., Buidina T.A. The relationship between quality of life indicators and characteristics of psychological status with 
clinical manifestations of metabolic syndrome. Quality Clinical Practice. 2006; 2: 19-23 (in Russian).

17. Пашковский В.Э., Софронов А.Г., Федоровский И.Д. Сравнительный анализ показателей социальной адаптации больных параноидный шизофренией с разной частотой госпитализаций. Социальная и клиническая психиатрия. 2017; 3: 1925. Pashkovsky V.E., Sofronov A.G., Fedorovsky I.D. Comparative analysis of indicators of social adaptation of patients with paranoid schizophrenia with different frequency of hospitalizations. Social and Clinical Psychiatry. 2017; 3: 19-25 (in Russian).

18. International Diabetes Federation. Clinical Guidelines Task Force. Global guideline for type 2 diabetes. Brussels: International Diabetes Federation; 2005.

19. Логвинович Г.В., Семке А.В. Первичные и вторичные нарушения адаптации при шизофрении. Томск : Изд-во Том. ун-та, 1995: 212. Logvinovich G.V., Semke A.V. Primary and secondary adaptation disorders in schizophrenia. Tomsk: Publishing House of the Tomsk University, 1995: 212 (in Russian).

20. Kay S.R., Opler L.A., Fiszbein A. The Positive and Negative Syndrome Scale (PANSS) for schizophrenia. Schizophr. Bull. 1987;13: 261-276. DOI: 10.1093/schbul/13.2.261

21. Мосолов С.Н. Шкалы психометрической оценки симптоматики шизофрении и концепция позитивных и негативных расстройств. М., 2001: 238. Mosolov S.N. Scales for psychometric assessment of schizophrenia symptoms and the concept of positive and negative disorders. Moscow, 2001: 238 (in Russian).
22. Бунак В.В. Антропометрия. Практический курс. М.: Учпедгиз, 1941: 367. Bunak V.V. Anthropometry. Practical course. Moscow : Uchpedgiz, 1941: 367 (in Russian).

23. Семке А.В., Федоренко О.Ю., Лобачева О.А., Рахмазова Л.Д., Корнетова Е.Г., Смирнова Л.П., Микилев Ф.Ф., Щигорева Ю.Г. Клинические, эпидемиологические и биологические предпосылки адаптации больных шизофренией как основа персонифицированного подхода к антипсихотической терапии. Сибирский вестник психиатрии и наркологии. 2015; 3(88): 19-25. Semke A.V., Fedorenko O.Yu., Lobacheva O.A., Rakhmazova L.D., Kornetova E.G., Smirnova L.P., Mikilev F.F., Shchigoreva Yu.G. Clinical, epidemiological and biological preconditions of adaptation of patients with schizophrenia as a basis for personalized approach to antipsychotic therapy. Siberian Herald of Psychiatry and Addiction Psychiatry. 2015; 3(88): 19-25 (in Russian).

24. Семке А.В., Ветлугина Т.П., Иванова С.А., Рахмазова Л.Д., Гуткевич Е.В., Лобачева О.А., Корнетова Е.Г. Биопсихосоциальные основы и адаптационно-компенсаторные механизмы шизофрении в регионе Сибири. Сибирский вестник психиатрии и наркологии. 2009; 5 (56): 15-20. Semke A.V., Vetlugina T.P., Ivanova S.A., Rakhmazova L.D., Gutkevich E.V., Lobacheva O.A., Kornetova E.G. Biopsychosocial bases and adaptive-compensatory mechanisms of schizophrenia in region of Siberia. Siberian Herald of Psychiatry and Addiction Psychiatry. 2009; 5 (56): 15-20 (in Russian).

Поступила в редакцию 30.04.2020 Утверждена к печати 02.09.2020

Хамина Виктория Владимировна, младший научный сотрудник отделения эндогенных расстройств НИИ психического здоровья Томского НИМЦ. ORCID ID 0000-0002-1001-5869. Author ID РИНЦ 938830. SPIN-код РИНЦ 9945-2016.

Хамина Виктория Владимировна, Vika.dubrovskaya.vd@gmail.com 
UDC 616.895.82-056.34:611.018.26:364.785.14:591.555.16

For citation: Khamina V.V. Features of adaptive abilities in patients with schizophrenia and metabolic syndrome. Siberian Herald of Psychiatry and Addiction Psychiatry. 2020; 3 (108): 51-56. https://doi.org/10.26617/1810-31112020-3(108)-51-56

\section{Features of adaptive abilities in patients with schizophrenia and metabolic syndrome}

\section{Khamina V.V.}

Mental Health Research Institute, Tomsk National Research Medical Center, Russian Academy of Sciences Aleutskaya Street 4, 634014, Tomsk, Russian Federation

\section{ABSTRACT}

The relevance of the work is determined by the demand for research to clarify and concretize the adaptive potential in schizophrenia with concomitant metabolic syndrome (MS) and the need to assess its relationship with the clinical and constitutional characteristics of patients. Objective: to study the features of the adaptive abilities of patients with schizophrenia, depending on the presence or absence of concomitant MS. Material and Methods. We examine 150 patients. Schizophrenia (F20) is diagnosed based on ICD-10 criteria. The diagnosis of MS is verified in accordance with the criteria of the International Diabetes Federation (2005). The adaptation of schizophrenic patients is assessed using the method of G.V. Logvinovich with the definition of the type of compensatory-adaptive defense of patients and the type of adaptation. Psychometric assessment of the clinical state is carried out using the PANSS scale. Discussion. Patients with schizophrenia and MS tend, like patients without MS, in most cases to form a type of compensatory-adaptive defense in the form of "shelter under care". Among the types of adaptation in patients with schizophrenia and concomitant MS, unfavorable ones (destructive and extraverted) prevail. The severity of the clinical condition in psychometric terms according to PANSS, as well as the constitutional features in patients with concomitant MS, do not differ depending on the type of social adaptation.

Keywords: schizophrenia, metabolic syndrome, types of adaptation, types of compensatory-adaptive defense, PANSS.

Received April 30.2020

Accepted September 02.2020

Khamina Viktoria V., junior researcher of the Endogenous Disorders Department, Mental Health Research Institute, Tomsk NRMC, Tomsk, Russian Federation. ORCID ID 0000-0002-1001-5869. Author ID RSCI 938830. SPIN-code RSCI 9945-2016.

Khamina Viktoria V., Vika.dubrovskaya.vd@gmail.com 